\title{
Repercusiones de la violencia intrafamiliar como factor de riesgo en el rendimiento académico
}

\section{Impact of domestic violence as a risk factor in academic performance}

\author{
Zoila Gladys Merino Armijos ${ }^{1, *}$, Sonia María Del Castillo Costa ${ }^{1, \dagger}$. \\ ${ }^{1}$ Universidad Nacional de Loja. \\ \{zgladys@hotmail.es, somadelcar@hotmail.com\}
}

Fecha de recepción: 30 de abril de 2017 — Fecha de aceptación: 31 de agosto de 2017

\begin{abstract}
Resumen-La violencia intrafamiliar es un fenómeno social que ha venido deteriorando las relaciones afectivas en todo el sistema familiar y su repercusión en el rendimiento académico y las relaciones interpersonales. Un problema actual y creciente entre los estudiantes, siendo de tal intensidad que ha provocado incidentes negativos, observándose esta problemática transversalmente en diversos contextos culturales y sociales; tiene como objetivo apreciar cómo se da la violencia intrafamiliar entre los escolares, los factores que están involucrados en estos eventos, así como también identificar algunas intervenciones que se han llevado a cabo para prevenir y tratar estas conductas, y los resultados obtenidos. Dentro de los factores que se relacionan con la violencia intrafamiliar están los de tipo individual, familiar, escolar y del ambiente. En las intervenciones realizadas han participado padres, profesores y/o alumnos(as), obteniéndose resultados positivos con un enfoque integral.
\end{abstract}

Palabras Clave-Agresión, violencia, aspectos psicológicos, aprendizaje, intervención.

\begin{abstract}
Domestic violence is a social phenomenon that has been deteriorating emotional relationships around the family system and its impact on academic performance and interpersonal relationships. A current and growing problem among students, being of such intensity that has caused negative incidents observed across this problem in different cultural and social contexts; aims to assess how domestic violence among schoolchildren is given, the factors that are involved in these events, as well as identify some interventions that have been carried out to prevent and treat these behaviors, and the results obtained. Among the factors that relate to domestic violence are the individual, family, school type and the environment. In interventions they have involved parents, teachers and / or students (as), yielding positive results with a comprehensive approach.
\end{abstract}

Keywords-Aggression, violence, psychological, learning, intervention

\section{INTRODUCCIÓN}

$\mathbf{U}$ n problema actual y creciente a nivel mundial y que también afecta a la educación ecuatoriana, es la agresión y violencia intrafamiliar observada entre los estudiantes, siendo de tal intensidad que ha provocado incidentes negativos en niños y adolescentes, como dificultad en el rendimiento académico y las interrelaciones de los estudiantes, que transversalmente se insertan en los diversos contextos culturales y sociales.

La violencia intrafamiliar se presenta en todas las formas de agresión que tienen lugar entre quienes existe un vínculo afectivo. Linda (2013) afirma que se ha convertido en un problema de riesgo a través de la historia de la humanidad y del mundo, enraizándose en América Latina como el mayor índice de violencia intrafamiliar.

El estudio realizado fue de tipo descriptivo, de corte transversal, se utilizó el método cuantitativo, porque se estudió las situaciones y eventos, además midió las causas y consecuencias de la violencia intrafamiliar en los estudiantes de octavo, noveno y décimo de Educación General Básica y de primero, segundo y tercero de bachillerato del Colegio "Vicente Anda Aguirre" de la ciudad de Loja.

\footnotetext{
*Licenciada en Ciencias de la Educación Mención CIencias Sociales, Máster en Educación y Desarrollo Social

${ }^{\dagger}$ Licenciada en Ciencias de la Educación en la Especialidad de Psicología infantil y Educación Parvularia, Máster en Educación Infantil
}

Los tipos de preguntas las respuestas fueron muy similares repitiéndose con frecuencia las siguientes frases para alumnos: "si" "no", "agresiones físicas", "insultos", "gritos", "cordial", "poco cordial", "regular", "bueno", "muy bueno"; padres de familia: "si", "no", "se reserva", "discuten frente a ellos", "se agreden", "se insultan"; docentes: "temor", "tolerancia", "estrictez", "diálogo", "hostil", "rebelde", "tranquilo", "violento", "expulsión”, “insultos”, "provocaciones".

Existen muchos estudios que informan los episodios de violencia intrafamiliar en hogares que están produciendo riesgos en los estudios, asociados a daños físicos y emocionales, estrés, desmotivación, ausentismo, e incluso efectos negativos en el rendimiento académico por estrés postraumático en los afectados. (S. Bales, 2000)

Frente a estos acontecimientos de violencia intrafamiliar se presenta el bajo rendimiento escolar y el deterioro de la relaciones interpersonales, lo cual resulta no indagar con mayor profundidad las causas que muchos de ellos producen mecanismo psíquicos que se ponen en juego ante la diversidad familiar, que afectan de forma diferenciada en aquellos procesos psicológicos superiores que se encuentran en estado de desarrollo.

Andrade (2016) en el Diario El Comercio hace referencia a que la violencia intrafamiliar es "toda acción u omisión protagonizada por los miembros que conforman el grupo familiar, este puede ser por afinidad, sangre o afiliación y que transforma en agresores las relaciones entre ellos causando 
daño físico, psicológico, sexual, económico o social a uno o varios de ellos".

La violencia intrafamiliar produce problemas de ajuste conductual, social y emocional, que se traducen en conducta antisocial en general, depresión y ansiedad y problemas en el Colegio. Los problemas de ajuste conductual y social se manifiestan en diferentes esferas, consumo de drogas, orientación académica y conducta criminal McGee and Newcomb (1992) en diferentes contextos como el "Colegio, casa, la comunidad en diferentes magnitudes que van desde expresiones leves a las más serias, Resnicow et al. (1995) mencionan que algunos autores argumentan que los problemas escolares, el consumo de drogas y la delincuencia se conoce que están altamente relacionados pero constituyen factores separados".

Instituto Nacional de Estadística y Censos (2011) menciona que: En base a la Primera Encuesta de Violencia de Género, el $60,6 \%$ de las mujeres en Ecuador ha vivido algún tipo de violencia. La violencia contra la mujer no tiene mayores diferencias entre zonas urbanas y rurales: en la zona urbana el porcentaje es de $61,4 \%$ y en la rural $58,7 \%$. (p. 1)

Diario la Hora (2012) La violencia intrafamiliar preocupa en la ciudad: En Loja, la Comisaria de la Mujer y la Familia atiende en promedio 150 denuncias mensuales de mujeres que fueron agredidas física o psicológicamente por sus cónyuges o convivientes; y 25 denuncias de hombres que también fueron víctimas de violencia o abandono por parte de sus esposas.

Las contravenciones de tercera y cuarta clase son juzgadas por la comisaría de Loja con sanciones de 2 a 4 días de prisión y multa de 7 ó 14 dólares; y de 5 a 30 días de prisión más una multa de 14 ó 28 dólares, respectivamente. Cuando hay presunción de delito la causa pasa a la Fiscalía.

Los fines de semana se detienen hasta 10 personas por haber sido sorprendidas en actos de violencia; casos de violencia registrados: Niños 31; Adolescentes 103; Mujeres 474; Hombres 46; Violencia intrafamiliar 497. (Diario la Hora, 2012)

Otro predictor importante que ayuda para el rendimiento académico es la autoconfianza. Marsh and Yeung (1997) han mostrado que ambos están relacionados, sin embargo, la autoconfianza se comporta en forma distinta en las diferentes asignaturas. Por ejemplo, de acuerdo con este autor, el rendimiento académico en matemáticas está sustancialmente correlacionado con la autoconfianza del estudiante sobre sus habilidades para esta asignatura, pero no con la autoconfianza para el inglés, mientras que la autoconfianza para inglés está sustancialmente relacionada con el rendimiento académico en esta asignatura, pero no en matemática.

La variable rendimiento escolar tiene diversas acepciones y dependiendo de la definición se emplean diferentes indicadores para medirla. Se puede referir al nivel de aprovechamiento que logra un alumno con respecto a los objetivos, prácticas y criterios educativos instituidos en determinado contexto sociocultural o al grado de logro de los objetivos establecidos en los programas escolares que involucra variables de orden cognitivo, volitivo y emocional.

Según Rossello (S.a) en su obra "Blanco y Negro) expresa: Vivenciar las relaciones interpersonales y la dinámica de lo que sucede en esta interacción, desarrollar destrezas para percatarse de la conducta de los demás y lo más importante aún, es desarrollar cierta medida de intromisión que haga comprender mejor individual y colectivamente. Recuperado. www.relacionessociales.html (p.23)

Un centro educativo con bachillerato es un contexto complejo que otorga al estudiante la oportunidad de adquirir no sólo conocimientos, sino actitudes, hábitos y estilos relacionales que pueden, incluso, neutralizar algunos efectos nocivos de un ambiente social y/o familiar desfavorecido, (Levinger, 1984). Hartup (1992) señala que: es un espacio que permite el ejercicio y el aprendizaje de las relaciones entre pares de forma privilegiada, contribuyendo no sólo al desarrollo cognitivo y social infantil, sino también a la eficacia interpersonal en la etapa adulta siendo uno de los mejores predictores de la adaptación adulta. En cambio el "fracaso social" escolar se puede manifestarse en rechazo, agresividad activa o pasiva, u otro tipo de problemática de adaptación, puede convertirse en un factor de riesgo para el desarrollo integral del niño.

Una relación interpersonal es una interacción recíproca entre dos o más personas; se trata de relaciones sociales que, como tales, se encuentran reguladas por las leyes e instituciones de la interacción social. En toda relación interpersonal interviene la comunicación, que es la capacidad de las personas para obtener información respecto a su entorno y compartirla con el resto de la gente.

\section{METOdología}

El estudio realizado es de tipo correlacional que se inicia con el desarrollo de un marco teórico, con intervención de las variables que fueron la violencia intrafamiliar, el rendimiento académico y las relaciones interpersonales de los estudiantes; como resultado de este trabajo, se transformó en una investigación explicativa y con los resultados obtenidos se explicó el problema propuesto, definiendo las variables correlacionadas.

Esta investigación se caracterizó por ser nacional y crítico, por su trascendencia de un estudio reflexivo, sistemático y metódico con la finalidad de ampliar conocimientos y emprender posibles soluciones mediante el procedimiento científico con el apoyo de técnicas específicas y obtener los objetivos planteados.

La población estudiada correspondió a 298 estudiantes del Colegio "Vicente Anda Aguirre" de la ciudad de Loja. Para determinar el tamaño de la muestra se prefijó un coeficiente confiabilidad del $91 \%$ y un error probable del $9 \%$, tomando el número de estudiantes que cursaron el octavo, noveno, décimo de básica, primero, segundo y tercero de bachillerato. Cuadro de universo de población estudiada de 298 personas. 
Tabla 1. Información obtenida Colegio "Vicente Anda Aguirre

\begin{tabular}{|l|c|c|c|}
\hline $\begin{array}{c}\text { Colegio "Vicente Anda } \\
\text { Aguirre" }\end{array}$ & Hombres & Mujeres & Total \\
\hline 8vo. Año de básica & 25 & 23 & 48 \\
\hline 9no. Año de básica & 27 & 24 & 51 \\
\hline 10mo. Año de básica & 29 & 22 & 51 \\
\hline 1ro. De bachillerato & 35 & 19 & 54 \\
\hline 2do. De bachillerato & 20 & 21 & 41 \\
\hline 3ro. De bachillerato & 30 & 23 & 53 \\
\hline TOTAL & $\mathbf{1 6 6}$ & $\mathbf{1 3 2}$ & $\mathbf{2 9 8}$ \\
\hline \multicolumn{4}{|r|}{ FUENTE: Secretaría Colegio "Vicente Anda Aguirre" } \\
\hline
\end{tabular}

De los 298 estudiantes se obtuvo una muestra representativa aplicando la siguiente fórmula:

$$
\mathrm{n}=\frac{\mathrm{N}}{\mathrm{e}^{2}(\mathrm{~N}-1)+1} \mathrm{n}=87
$$

Obtenido la muestra equivalente a 87 estudiantes se procedió a la distribución al azar.

\begin{tabular}{|c|c|c|c|c|}
\hline \multirow{7}{*}{ 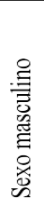 } & Estratos & Población & Proporción & Total \\
\hline & $8^{\circ}$ Año de básica & 25 & 8,39 & 7 \\
\hline & $9^{\circ}$ Año de Básica & 27 & 9,00 & 8 \\
\hline & $10^{\circ}$ Año de básica & 29 & 9,73 & 8 \\
\hline & $1^{\circ}$ de bachillerato & 35 & 11,74 & 10 \\
\hline & $2^{\circ}$ de bachillerato & 20 & 6,71 & 6 \\
\hline & $3^{\circ}$ de bachillerato & 30 & 10,07 & 9 \\
\hline \multirow{7}{*}{ 总 } & $8^{\circ}$ de básica & 23 & 7,17 & 7 \\
\hline & $9^{\circ}$ de básica & 24 & 8,05 & 7 \\
\hline & $10^{\circ}$ de básica & 22 & 7,38 & 6 \\
\hline & $1^{\circ}$ de bachillerato & 19 & 6,36 & 6 \\
\hline & $2^{\circ}$ de bachillerato & 21 & 7,05 & 6 \\
\hline & $3^{\circ}$ de bachillerato & 23 & 7,72 & 7 \\
\hline & TOTAL & 298 & 1,00 & 87 \\
\hline
\end{tabular}

Confirmada la muestra, se aplicaron las técnicas con la finalidad de recolectar la información sobre la violencia intrafamiliar que afectan al rendimiento académico y las relaciones interpersonales. Recopilación de bibliografía: fue utilizada durante el proceso de investigación a través de libros, folletos, revistas, informes.

Entrevistas: se entrevistó a estudiantes, profesores y padres de familia con la finalidad de conocer la metodología que utilizan los maestros en cuanto a: el dictado, resúmenes, material didáctico, práctica de valores, responsabilidad y forma de evaluar.

Encuestas: se aplicaron a estudiantes, profesores y padres de familia para conocer: rol que cumplen, desenvolvimiento académico, actuación estudiantil, relaciones interpersonales, rendimiento académico, integración de los padres.

Instrumentos: Para la medición de las variables bajo estudio se utilizaron tres encuestas las cuales se describen a continuación.

1. Encuesta a estudiantes: fue elaborado por la autora y está diseñada de forma semiestructurada con preguntas abiertas y cerradas que permiten explorar los siguientes aspectos: i) Comportamiento violento en el colegio, ii) incremento de violencia en el centro educativo, iii) violencia en el hogar, iv) castigo que aplican sus padres, v) ambiente en el aula de clases, vi) violencia en el hogar, vii) rendimiento académico, viii) relaciones interpersonales, ix) violencia en el hogar incide en el rendimiento académico. El análisis factorial de este cuestionario arrojó tres factores de violencia intrafamiliar, rendimiento académico y relaciones interpersonales que en conjunto explican el $95 \%$ de la varianza total.

2. Encuesta a padres de familia: se utilizó un instrumento diseñado por la autora de forma semiestructurada con cinco preguntas que permitió conocer los aspectos siguientes: i) tiempo que dedica a sus hijos para conversar y ayuda a las tareas escolares, ii) conducta hostil y agresiva de sus hijos en el hogar, iii) relación conyugal en el hogar, iv) desarrollo creatividad de su hijo, v) amigos de su hijo. El análisis factorial de este cuestionario arrojó tres factores de diálogo y apoyo, conducta de su hijo en el hogar y relación conyugal que en conjunto explican el $95 \%$ de la varianza total.

3. Encuesta a profesores:se utilizó un instrumento diseñado por la autora de forma semiestructurada consta de cinco item los aspectos siguientes: i) ejercicio de la autoridad del plantel, ii) comportamiento de los estudiantes, iii) castigo de los alumnos violentos, iv) violencia intrafamiliar incide en relaciones interpersonales, v) demostración de la violencia en el colegio. El análisis factorial de este cuestionario arrojó tres factores de autoridad y comportamiento, castigo por la violencia y relación interpersonal, que en conjunto explican el $95 \%$ de la varianza total.

Procedimiento.-En primer término fue necesario realizar la selección del establecimiento educativo y participantes. Se estableció un proceso de muestreo accidental, para el que se determinó algunos parámetros que se describen a continuación: i) La muestra obtenida se la investigó en un colegio Fiscomisional por encontrarse estudiantes de diferentes estratos sociales y por estar regentado por una comunidad clerical y administrada por un Proyecto Educativo y Código de Convivencia en base a la Filosofía y normas religiosas de la Federación de Establecimientos de Educación Católica de Loja.

Seguidamente, con la finalidad de establecer la funcionalidad y pertinencia de los instrumentos, se llevó a cabo una encuesta, demostrando evidencia de la necesidad de conocer los problemas de la violencia intrafamiliar y su incidencia en el rendimiento académico y la relaciones interpersonales de los estudiantes, la mejor hora para aplicar los cuestionarios es durante el primer período de clases, porque los estudiantes están con mayor disponibilidad de responder, que si se hiciera a mitad de la jornada o al final.

Para la etapa de recolección de los datos, el tiempo de aplicación de los instrumentos sumó en total tres horas, por lo cual se solicitaron los permisos necesarios en el plantel, para aplicar las encuestas durante dos días de una hora y media cada día, con el fin de evitar la fatiga en los estudiantes.

El segundo día se reunió a los padres de familia para 
aplicarles la encuesta para conocer los tres factores de diálogo y apoyo, conducta de su hijo en el hogar y relación conyugal. En el tercer día se aplicó la encuesta a los profesores en el horario de receso con la finalidad de conocer autoridad y comportamiento, castigo por la violencia y relación interpersonal.

Finalmente, los datos se ingresaron a un ordenador a través de un lector óptico, que permite cotejar cada cuestionario ingresado de manera física, de tal forma que se puedan corregir los errores de lectura o de respuesta.

Después se exportaron las bases de datos Impacto de la violencia intrafamiliar, el rendimiento académico y las relaciones interpersonales al programa SPSS en donde se procedió a ordenarlas, calificarlas y codificarlas para los análisis de confiabilidad, y se establecieron valores típicos (Z) para el análisis de correlación.

El estudio estuvo basado en un enfoque cuantitativo que permitió determinar las características externas generales de una población, basándose en la observación de muchos casos similares de la misma. La investigación fue de tipo correlacional, para el análisis de los datos se utilizaron estadísticas descriptivas; al formular una hipótesis es volver a evaluar el planteamiento del problemas y para la fase comprobatoria de las hipótesis fue necesario la intervención y apoyo de la prueba Chi-Cuadrado. Para el análisis de regresión múltiple se utilizó el método Enter mediante el cual se ingresaron todas las variables de control para determinar el aporte de cada una de ellas al rendimiento académico y a las relaciones interpersonales.

\section{RESUlTADOS}

En esta sección se presenta la información obtenida a partir de la codificación y análisis de los datos recolectados en la aplicación de las encuestas.

El primer objetivo específico,es determinar las causas del comportamiento violento de los estudiantes.

Tabla 2. Comportamiento violento en la institución educativa

\begin{tabular}{|l|c|c|}
\hline \multicolumn{1}{|c|}{ Variable } & f & $\%$ \\
\hline Si & 63 & $72,42 \%$ \\
\hline No & 24 & $27.58 \%$ \\
\hline TOTAL & $\mathbf{8 7}$ & $\mathbf{1 0 0}$ \\
\hline
\end{tabular}

FUENTE: Secretaría Colegio "Vicente Anda Aguirre"

ELABORACIÓN: Zoila Gladys Merino Armijos.

Gráfico 2.Comportamiento violento en la institución educativa

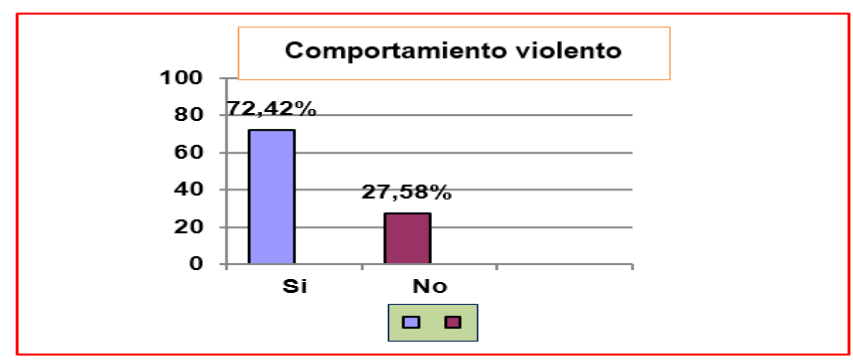

FUENTE: Secretaría Colegio "Vicente Anda Aguirre" ELABORACIÓN: Zoila Gladys Merino Armijos.
En la tabla y gráfico 63 estudiantes que equivalen al 72,42\% manifiestan que en el centro educativo se presentan casos de comportamiento violento. Lo cual expresa que la Violencia Intrafamiliar detectada se caracteriza en mayor medida la violencia por gritos, insultos, críticas, humillaciones, entre otras.

Tabla 3. Violencia incrementada en el Colegio

\begin{tabular}{|l|c|c|}
\hline Variable & f & \% \\
\hline $\mathrm{Si}$ & 87 & $100 \%$ \\
\hline No & 0 & $0 \%$ \\
\hline TOTAL & $\mathbf{8 7}$ & $\mathbf{1 0 0}$ \\
\hline
\end{tabular}

FUENTE: Secretaría Colegio "Vicente Anda Aguirre" ELABORACIÓN: Zoila Gladys Merino Armijos.

Gráfico 3.Violencia incrementada en el Colegio

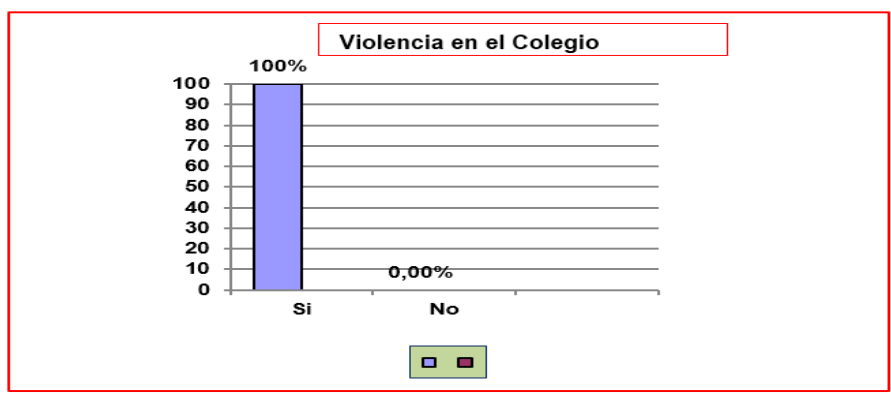

FUENTE: Secretaria Colegio "Vicente Anda Aguirre"

EL ABOR ACIÓN Zoila Gladys Merino Armijos.

Para el $100 \%$ de alumnos mencionan que en el centro educativo se ha incrementado actitudes de violencia. Los datos antes expuestos nos hace reflexionar que este fenómeno no es concientizado por los(as) entrevistado(os); las manifestaciones de la violencia son percibidas como algo normal y cotidiano, que forma parte del sistema de relación familiar habitual.

Tabla 4. Violencia que se presentan en el hogar

\begin{tabular}{|c|c|c|}
\hline Variable & $\mathrm{f}$ & $\%$ \\
\hline $\mathrm{Si}$ & 70 & $80,46 \%$ \\
\hline No & 17 & $19,54 \%$ \\
\hline TOTAL & $\mathbf{8 7}$ & $\mathbf{1 0 0}$ \\
\hline
\end{tabular}

FUENTE: Secretaría Colegio "Vicente Anda Aguirre" ELABORACIÓN: Zoila Gladys Merino Armijos

Gráfico 4.Violencia que se presentan en el hogar

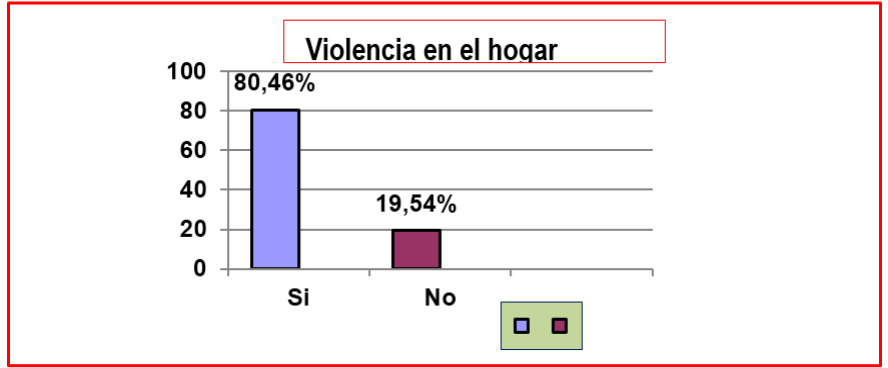

FUENTE: Secretaría Colegio "Vicente Anda Aguirre" ELABORACIÓN: Zoila Gladys Merino Armijos. 
Suman 70 alumnos afirman que en sus hogares si existe la violencia familiar, generado por sus padres, se caracteriza en mayor medida por gritos, insultos, críticas, humillaciones, entre otras.

Tabla 5. Ambiente violento en el hogar influye el comportamiento agresivo en el Colegio

\begin{tabular}{|c|c|c|}
\hline Variable & f & $\%$ \\
\hline $\mathrm{Si}$ & 87 & $100 \%$ \\
\hline No & 0 & $0 \%$ \\
\hline TOTAL & $\mathbf{8 7}$ & $\mathbf{1 0 0}$ \\
\hline \multicolumn{3}{|c|}{ FUENTE: Secretaria Colegio "Vicente Anda Aguirre" } \\
ELABORACION: Zoila Gladys Merino Armijos
\end{tabular}

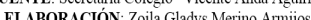

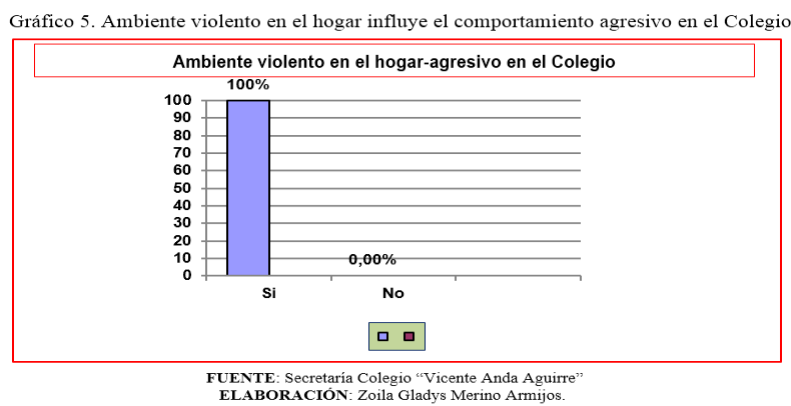

El $100 \%$ de estudiantes, manifiestan que en sus hogares reina la violencia, lo cual provoca que los hijos sean violentos y prevalezca el dominio a otros alumnos más débiles del centro educativo sin medir las consecuencias.

Tabla 6. Hijos con conducta hostil y agresiva en el hogar

\begin{tabular}{|c|c|c|}
\hline Variable & f & $\%$ \\
\hline Si & 25 & $71,43 \%$ \\
\hline No & 10 & $28,57 \%$ \\
\hline TOTAL & $\mathbf{3 5}$ & $\mathbf{1 0 0}$ \\
\hline
\end{tabular}

FUENTE: Secretaría Colegio "Vicente Anda Aguirre" ELABORACIÓN: Zoila Gladys Merino Armijos

Gráfico 6. Hijos con conducta hostil y agresiva en el hogar

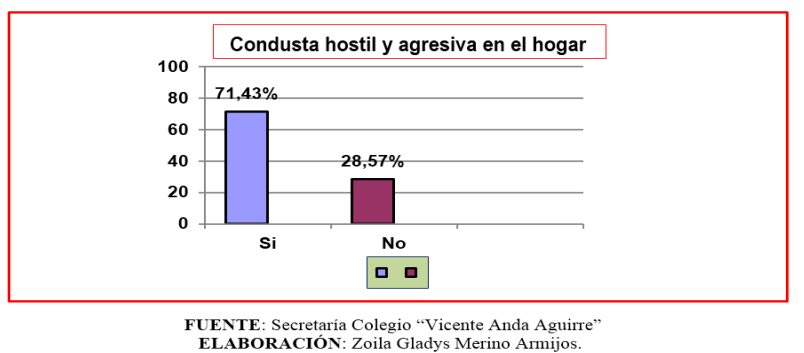

TLNEORACIÓ : Zoila Gladys Merino Armijos.

Con respecto a esta pregunta, los padres de familia exponen que la mayoría de los menores demuestran una actitud agresiva y hostil dentro del hogar en un total de 25 que representan el $71,42 \%$. En estas familias se da una expresión desfavorable de armonía, cohesión, afectividad, comunicación, entre otras, demostrando la disfuncionabilidad familiar por la presencia de violencia física y psicológica en estos hogares.
El tercer objetivo específico,es precisar las relaciones interpersonales de los estudiantes.

Tabla 7. Relaciones interpersonales de los estudiantes en el Colegio

\begin{tabular}{|c|c|c|}
\hline Variable & f & $\%$ \\
\hline Mala & 50 & $57,48 \%$ \\
\hline Regular & 26 & $29,88 \%$ \\
\hline Buena & 11 & $12,64 \%$ \\
\hline TOTAL & $\mathbf{8 7}$ & $\mathbf{1 0 0}$ \\
\hline
\end{tabular}

FUENTE: Secretaría Colegio "Vicente Anda Aguirre" ELABORACIÓN: Zoila Gladys Merino Armijos

Gráfico 7.Relaciones interpersonales de los estudiantes en el Colegio

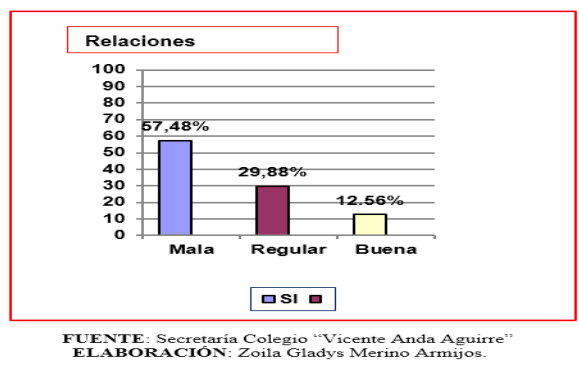

El 50 estudiantes representan el 57,48\% de investigados manifiestan que tienen malas relaciones en el Colegio incidiendo en su desarrollo personal; 26 que es el $29,88 \%$ dicen que sus relaciones personales son regulares porque se interactúa con todos los compañeros; 11 estudiantes que es el 12,64\% manifiestan que sus relaciones son buenas, porque evitan tener problemas con los alumnos conflictivos y por temor a ser llamados la atención de las autoridades del Colegio.

\section{DisCuSión DE RESUltados}

El propósito de esta investigación fue analizar los efectos de la violencia intrafamiliar en estudiantes del Colegio "Vicente Anda Aguirre" de la ciudad de Loja, confirmando sus resultados una vez más la hipótesis de que la violencia intrafamiliar incide en el rendimiento académico y el desarrollo de las relaciones interpersonales de los estudiantes. Un objetivo general fue investigar cómo afecta la violencia intrafamiliar en el rendimiento académico y en las relaciones interpersonales de los/las estudiantes. Se comprobó la hipótesis planteada en el sentido que está generando un factor de violencia intrafamilia que a su vez genera problemas en el rendimiento académico $\mathrm{y}$ en las relaciones interpersonales de tipo social, emocional y conductual en los estudiantes. Sin embargo, los efectos que se producen en cada uno de estos ajustes son diferentes. La violencia muestra un efecto directo en el rendimiento académico y en las relaciones interpersonales produciendo ansiedad y comportamientos violentos, con efecto indirecto hacia los problemas escolares. Algunos autores han propuesto a manera de hipótesis que la violencia intrafamiliar es de ajuste emocional; sin embargo, esta investigación demostró que la violencia intrafamiliar incide en el rendimiento académico y las relaciones interpersonales de los estudiantes.

En el presente estudio, las formas de violencia intrafamiliar se producen de forma directa e indirecta, produciendo 
problemas psicosociales de los alumnos, manifestándose éstos como problemas de conducta, alteraciones psicológicas, comportamiento violento y malas relaciones interpersonales. Los resultados son consistentes con otros estudios que muestran una asociación entre la violencia intrafamiliar y diversos problemas de rendimiento académico. Estos resultados coinciden con los hallazgos de Strauss and Colby (2001) Strauss (2002), quienes mostraron correlaciones significativas entre maltrato en el hogar y rendimiento académico.

De igual forma el maltrato intrafamiliar que es nocivo al estado emocional y físico de los estudiantes que son agredidos dentro del hogar, en la actualidad es un factor de riesgo en el rendimiento académico de los sujetos que formaron parte de esta investigación. Estos resultados concuerdan con los hallazgos de investigaciones realizadas por Strauss (2002) se encontró una relación inversa entre el maltrato de los padres y el rendimiento académico de los hijos.

Otro hallazgo importante en esta investigación fue que la violencia se ha incrementado en el centro educativo el $100 \%$ con actitudes violentas de los estudiantes, indicando que los episodios de agresión doméstica prácticamente afloran contra los compañeros indefensos. Esto coincide con lo encontrado en el Colegio del Bachillerato del Instituto Superior Vicente León de la ciudad de Latacunga que se identifica la agresión física y verbal es el tipo de violencia intrafamiliar que se presenta con mayor frecuencia; se caracteriza por la formación educativa de los padres que se encuentra del nivel primario hacia abajo; además la prepotencia, el alcoholismo y la mala situación económica desencadenan las discusiones y agresiones que recaen en los jóvenes estudiantes. (Rivadeneira Miño, 2012)

Un ambiente violento en el hogar influye notablemente en el comportamiento agresivo de los estudiantes en el Colegio, en este estudio se logró detectar que el $100 \%$ de estudiantes mencionan que en sus hogares la violencia es permanente, lo cual provoca que los hijos sean violentos en el centro educativo. Esto concuerda con estudios anteriores que muestran que la violencia intrafamiliar se relaciona con altos niveles de esas alteraciones violentas. (Japa Banegas, 2015)

La violencia intrafamiliar incide en el rendimiento escolar de los estudiantes, lo que refleja y repercute en el rendimiento académico y el buen desempeño de las actividades curriculares, como efecto de esta violencia se manifiestan a través de problemas de conducta y psicológicos. Resultados que concuerdan con la investigación realizada en la Universidad de Cuenca "Causas y consecuencias de la violencia intrafamiliar en los niños de segundo a séptimo año de básica, Unidad Educativa Comunitaria Rumiñahui" al exponer que Las características de niños que sufren algún tipo de violencia de la Unidad Educativa Comunitaria "Rumiñahui" son: incertidumbre, indecisión, ansiedad, descontrol, agresividad con el $65.2 \%$; terquedad y mal humor con un $33.7 \%$; comportamientos maniáticos y rasgos psicóticos con el $30.4 \%$; egoísmo con el0.7\%; timidez con el $18.5 \%$ y extroversión con el $16.3 \%$. (Cusco Quito et al., 2014)

Los resultados aquí expuestos evidencian que las relaciones interpersonales de los estudiantes en el Colegio el 57,48\% es mala; el $29,88 \%$ es regular y el $12,64 \%$ es buena. Resultados que concuerdan con la tesis de investigación realizada en la
Universidad Técnica de Ambato, en la cual se determina que las relaciones interpersonales influyen más de manera negativa, que positiva en el rendimiento académico de inglés de los estudiantes de segundo de Bachillerato de la Especialidad Físico Matemático del Colegio Nacional Experimental Salcedo. (Crespo Acuña, 2013)

\section{Conclusiones}

- La investigación ha determinado que 63 estudiantes $(72,42 \%)$, que corresponden la mayoría afirman que se han dado casos de violencia en esta institución educativa, y 24 estudiantes $(27,58 \%)$ refieren no conocer que se hayan dado un hecho de este tipo. Concluyendo además que las situaciones de violencia existentes entre compañeros se dan en el establecimiento educativo.

- Los 87 estudiantes encuestados (100\%), observan comportamientos violentos en diferentes ámbitos de la sociedad, hogar, colegio, lo cual se refleja en la afectación que tienen en el rendimiento escolar y las relaciones interpersonales.

- El total de 70 alumnos encuestados (80,45\%), respondieron que en sus hogares si se han presentado casos de violencia, especialmente entre los padres, generadas por aspectos económicos, alcoholismo, desempleo, desorganización familiar, y 17 estudiantes $(19,54 \%)$ manifiestan no haber tenido estas situaciones de violencia.

- El $100 \%$ de estudiantes, manifiestan que en sus hogares reina la violencia, lo cual provoca que los hijos sean violentos y prevalezca el dominio a otros alumnos más débiles del centro educativo sin medir las consecuencias.

- Con respecto a esta pregunta, los padres de familia exponen que la mayoría de los menores demuestran una actitud agresiva y hostil dentro del hogar en un total de 25 que representan el 71,42\%. En estas familias se da una expresión desfavorable de armonía, cohesión, afectividad, comunicación, entre otras, demostrando la disfuncionalidad familiar por la presencia de violencia física y psicológica en estos hogares.

- 50 estudiantes representan el 57,48\% de investigados manifiestan que tienen malas relaciones en el Colegio incidiendo en su desarrollo personal; 26 que es el 29,88\% dicen que sus relaciones personales son regulares porque se interactúa con todos los compañeros; 11 estudiantes que es el 12,64\% manifiestan que sus relaciones son buenas, porque evitan tener problemas con los alumnos conflictivos y por temor a ser llamados la atención de las autoridades del Colegio.

\section{RECOMENDACIONES}

- A las autoridades nacionales fortalecer las políticas de Estado, a través de instituciones con el fin de proteger los derechos básicos de los adolescentes en cuanto a la supervivencia, educación y protección frente a la explotación y malos tratos, es decir haciendo cumplir lo que dicta el Código de la Niñez y la Adolescencia en lo referente a la Protección contra el Maltrato, Abuzo, Explotación Sexual, Tráfico, Perdida de Niños y Adolescentes, en los 
artículos 67 al 79; y en lo referente a la Ley contra la Violencia a la Mujer.

- Que los centros educativos organicen y ejecuten charlas constructivas, con la finalidad de modificar la conducta, y realizar talleres con sus docentes para poder atender a los estudiantes que exhiben actitudes violentas, para lograr un buen desarrollo social, reforzando el aspecto afectivo y frenar la incidencia de la violencia que afecta en el rendimiento académico y en las relaciones interpersonales de los estudiantes.

- Que en los hogares de los alumnos investigados, se brinde más atención por parte del servicio social de la institución educativa del Consejo Nacional de la Niñez y Adolescencia, organizaciones que trabajan en la protección del niño y la familia, a fin de que el desarrollo social y afectivo de los adolescentes alcance niveles de madurez y se promueva el diálogo, para que así se logre mejorar la comprensión entre los miembros de la familia.

- Efectuar reuniones los maestros y padres de familia de orientación y motivación con la finalidad de elevar el respeto entre los miembros de la familia, y ayudar a resolver los problemas del hogar, con la finalidad de eliminar todo tipo de violencia.

- Que la educación que se imparte en las instituciones educativas debe estar centrada en la convivencia, el respeto mutuo, la igualdad y la colaboración de todos los actores, como son: Maestros, padres de familia, estudiantes y comunidad en general.

- Organizar talleres, cursos y seminarios de capacitación a todo el personal de los centros educativos y comunidad en general, sobre la erradicación de la violencia intrafamiliar y la existente entre estudiantes.

\section{Bibliografía}

Andrade, J. (2016). En la revista Familia, Diario el Comercio. Crespo Acuña, D. F. (2013). Las relaciones interpersonales y su influencia en el rendimiento académico de inglés de los estudiantes de segundo de bachillerato de la especialidad físico matemático del colegio nacional experimental "salcedo" en el periodo académico 2009-2010. B.S. thesis.

Cusco Quito, M. E., Aucapiña, D., Catalina, M., Saeteros, S., and Imelda, F. (2014). Causas y consecuencias de la violencia intrafamiliar en niños de segundo a séptimo año de básica, unidad educativa comunitaria rumiñahui. quilloac 2014. B.S. thesis.

Diario la Hora (2012). La Violencia Intrafamiliar. La Hora Noticias de Ecuador, sus provincias y el mundo.

Instituto Nacional de Estadística y Censos (2011). Primera Encuesta de Violencia de Género.

Japa Banegas, I. K. (2015). Violencia doméstica hacia la mujer. B.S. thesis.

Levinger, B. (1984). School feeding programmes: myth and potential. Prospects, 14(3):369-376.

Linda, K. (2013). Violencia Intrafamiliar.

Marsh, H. W. and Yeung, A. S. (1997). Academic self-concept and achievement. American Educational Research Journal, 34(4):691-720.
McGee, L. and Newcomb, M. D. (1992). General deviance syndrome: expanded hierarchical evaluations at four ages from early adolescence to adulthood. Journal of consulting and clinical psychology, 60(5):766.

Resnicow, K., Ross-Gaddy, D., and Vaughan, R. D. (1995). Structure of problem and positive behaviors in african american youths. Journal of Consulting and Clinical Psychology, 63(4):594.

Rivadeneira Miño, F. O. (2012). Violencia intrafamiliar y sus efectos en el rendimiento académico de los estudiantes de bachillerato del instituto vicente león de latacunga, período 2010-2011. Master's thesis, Universidad de Guayaquil. Facultad de Ciencias Médicas.

S. Bales, Susan J. Eklund, C. F. S. S. (2000). Children's perceptions of elders before and after a school-based intergenerational program. Educational Gerontology, 26(7):677689.

Strauss, M. (2002). Corporal punishment and academic achievement scores of young children: a longitudinal study. Recuperado el, 23.

Strauss, M. and Colby, J. (2001). Corporal punishment by mothers and academic achievement scores of young children: a longitudinal study. Family Research Laboratory New Hampshire University. 